\title{
Neuronal Migration Disorders
}

National Institute of Neurological Disorders and Stroke (NINDS)

\section{Source}

National Institute of Neurological Disorders and Stroke (NINDS). Neuronal Migration

Disorders Information Page.

Neuronal migration disorders (NMDs) are a group of birth defects caused by the abnormal migration of neurons in the developing brain and nervous system. In the developing brain, neurons must migrate from the areas where they are born to the areas where they will settle into their proper neural circuits. Neuronal migration, which occurs as early as the second month of gestation, is controlled by a complex assortment of chemical guides and signals. When these signals are absent or incorrect, neurons do not end up where they belong. This can result in structurally abnormal or missing areas of the brain in the cerebral hemispheres, cerebellum, brainstem, or hippocampus. The structural abnormalities found in NMDs include schizencephaly, porencephaly, lissencephaly, agyria, macrogyria, polymicrogyria, pachygyria, microgyria, micropolygyria, neuronal heterotopias (including band heterotopia), agenesis of the corpus callosum, and agenesis of the cranial nerves. Symptoms vary according to the abnormality, but often feature poor muscle tone and motor function, seizures, developmental delays, impaired cognitive development, failure to grow and thrive, difficulties with feeding, swelling in the extremities, and a smaller than normal head. Most infants with an NMD appear normal, but some disorders have characteristic facial or skull features that can be recognized by a neurologist. Several genetic abnormalities in children with NMDs have been identified. Defects in genes that are involved in neuronal migration have been associated with NMDs, but the role they play in the development of these disorders is not yet wellunderstood. More than 25 syndromes resulting from abnormal neuronal migration have been described. Among them are syndromes with several different patterns of inheritance; genetic counseling thus differs greatly between syndromes. 